\title{
Static structure and dynamics of the liquid Li-Na and Li-Mg alloys
}

\author{
M. Canales \\ Departament de Física i Enginyeria Nuclear, Universitat Politècnica de Catalunya, 08028 Barcelona, Spain \\ D. J. González and L. E. González \\ Departamento de Física Teórica, Facultad de Ciencias, Universidad de Valladolid, 47011 Valladolid, Spain \\ J. A. Padró \\ Departament de Física Fonamental, Universitat de Barcelona, 08028 Barcelona, Spain
}

(Received 20 April 1998; revised manuscript received 1 July 1998)

\begin{abstract}
We present calculations for the static structure and ordering properties of two lithium-based $s-p$ bonded liquid alloys, $\mathrm{Li}-\mathrm{Na}$ and $\mathrm{Li}-\mathrm{Mg}$. Our theoretical approach is based on the neutral pseudoatom method to derive the interatomic pair potentials, and on the modified-hypernetted-chain theory of liquids to obtain the liquid static structure, leading to a whole combination that is free of adjustable parameters. The study is complemented by performing molecular dynamics simulations which, besides checking the theoretical static structural results, also allow a calculation of some dynamical properties. The obtained results are compared with the available experimental data. [S1063-651X(98)07110-4]

PACS number(s): 61.25.Mv, 61.20.Ja
\end{abstract}

\section{INTRODUCTION}

The combination of the pseudopotential perturbation theory and modern accurate liquid state theories and/or computer simulation techniques has led to a remarkable progress in the theoretical description of the static, thermodynamic, and dynamic properties of simple ( $s-p$ bonded) liquid metals $[1,2]$. This success has basically relied on accurate pseudopotentials, which can be derived from first principles, and the use of the homogeneous electron gas as the reference state for the perturbative calculation of the energy of the system.

However, when it comes to a study of their liquid alloys, the situation is rather different. Although much work has been devoted to the study of the structural and thermodynamic properties of liquid binary alloys by using semiempirical models $[1,3]$, their study from a more fundamental level has been more infrequent. Some reasons may explain this situation. First, the environement of an ion in an alloy can be very different from that of a free atom, where standard pseudopotentials are constructed; therefore, the pseudopotential is likely to change and transferability problems may arise. Second, the pseudopotential may cease to be weak, and consequently perturbation theory will no longer be valid. Third, covalent/ionic tendencies may appear in the alloy, and the use of the homogeneous electron gas as a reference system will not be appropriate. The second and third problems may be overcome by resorting to first principles molecular dynamics, which uses density functional theory (DFT) instead of perturbation theory, although this approach does usually rely on free-atom computed pseudopotentials. However, even in those cases where the second and third problems above do not apply, still the main problem hinges on deriving suitable pseudopotentials from which reliable interatomic forces between the alloy components can be constructed.

In this paper we study two Li-based liquid alloys, namely, $\mathrm{Li}_{x} \mathrm{Na}_{1-x}$ and $\mathrm{Li}_{x} \mathrm{Mg}_{1-x}$, which may be considered as simple liquid alloys where the nearly-free-electron (NFE) theory holds, and, therefore, the pseudopotential perturbation theory can be applied. In fact, for both alloys the valence and electronegativity differences are small $(0.1$ and 0.2 , respectively, on the Pauling scale); moreover, there are some experimental measurements which suggest a NFE behavior $[1,4]$. Specifically, the present work focuses mainly on a study of the static structural features of both liquid alloys. This is mainly motivated by the availability of experimental results for their static structure, which show a completely different behavior: Li-Na is a typical phase separating system [5], whereas Li-Mg exhibits weak heterocoordination tendencies [4].

Our study comprises two steps: (i) the construction of pseudopotentials from which, by applying linear response theory, the effective interatomic pair potentials in the liquid alloy are derived; and (ii) the calculation of the liquid structure from these interatomic interactions, by using both a liquid state theory and molecular dynamics (MD) simulations.

The construction of the effective interatomic pair potentials has been carried out within the framework of the neutral pseudoatom (NPA) method [6-8], which we have already successfully applied to study the structural and thermodynamic properties of the liquid alkali and alkaline-earth metals $[9,10]$, as well as the structural features of the liquid $\mathrm{Na}_{x} \mathrm{~K}_{1-x}$ alloy [11]. The philosophy of the NPA method is similar to that of the pseudopotential theory, and so is its domain of applicability. In conjunction with DFT, it is entirely $a b$ initio, and has the advantage of handling true densities rather than pseudodensities. In this way, we obtain effective interatomic potentials in the alloy which are free of adjustable parameters, and using the atomic numbers of the components as the only input data.

The calculation of the static liquid structure is carried out by resorting to the modified-hypernetted-chain (MHNC) theory of liquids; this theory belongs to the new generation of accurate integral-equation theories of liquids [12]. Its ap- 
plication to various one-component fluids-ranging from simple model potentials to the liquid alkali and alkali-earth metals - as well as binary mixtures interacting via LennardJones potentials, has yielded excellent results for both the structural and thermodynamic properties. Finally, for both alloys at some specific concentrations, their liquid structure has also been computed by MD simulations which, besides checking the accuracy of the MHNC theory, also allows one to study some time dependent properties.

The paper is organized as follows. In Sec. II we briefly describe the NPA method, which is the basis to calculate the effective interatomic pair potentials used in the calculations, and we also summarize the main features of the liquid state theory used. Section III gives a brief description for the technical details of the MD simulations, whereas in Secs. IV and $\mathrm{V}$ we present and discuss the results obtained for the structural and dynamical features of both liquid alloys. Finally, we complete the paper in Sec. VI.

\section{THEORETICAL FORMALISM}

\section{A. Effective interatomic potentials: NPA method}

A simple liquid metallic alloy $A_{x} B_{1-x}$ can be regarded as an assembly of $A$ - and $B$-type bare ions with charges $Z_{v}^{A}$ and $Z_{v}^{B}$, respectively, embedded in a conduction electron gas of mean density $\bar{n}=\rho\left[x Z_{v}^{A}+(1-x) Z_{v}^{B}\right]$, where $\rho$ is the total ionic number density and $x$ is the concentration of the $A$-type component. Moreover, the ions attract the valence electrons which pile up around them, thus screening the ionic potentials and leading to effective interactions between the ions. Now we briefly describe the method for obtaining the interatomic pair potentials, and for additional details we refer to Refs. [6,7,10].

The calculation of the effective interatomic pair potentials involves two distinct steps. First, the valence electronic densities induced by each type of ion when embedded in an homogeneous electron gas with density $\bar{n}$ are obtained by the NPA method; then, in the second step we construct $A$ - and $B$-type effective local pseudopotentials which, within linear response theory (LRT), reproduce the corresponding induced electronic densities as obtained in the first step. Finally, from both pseudopotentials the effective interatomic pair potentials are obtained. Here, we briefly discuss both steps.

Within the NPA method, it is assumed that the total electronic density $n_{e}(r)$ of the $A_{x} B_{1-x}$ alloy can be decomposed as a sum of single site, structure independent and localized electronic densities $n^{(A)}(r)$ and $n^{(B)}(r)$, which follow the ions in their movement, so that (Hartree atomic units will be used through the paper)

$$
\begin{aligned}
n_{e}(r)= & \sum_{i} n^{(A)}\left(\left|\mathbf{r}-\mathbf{R}_{i}^{(A)}\right|\right)+\sum_{j} n^{(B)}\left(\left|\mathbf{r}-\mathbf{R}_{j}^{(B)}\right|\right) \\
= & \sum_{i}\left[n_{c}^{(A)}\left(\left|\mathbf{r}-\mathbf{R}_{i}^{(A)}\right|\right)+n_{v}^{(A)}\left(\left|\mathbf{r}-\mathbf{R}_{i}^{(A)}\right|\right)\right] \\
& +\sum_{j}\left[n_{c}^{(B)}\left(\left|\mathbf{r}-\mathbf{R}_{j}^{(B)}\right|\right)+n_{v}^{(B)}\left(\left|\mathbf{r}-\mathbf{R}_{j}^{(B)}\right|\right)\right],
\end{aligned}
$$

where $\mathbf{R}_{i}^{(A)}$ and $\mathbf{R}_{j}^{(B)}$ denote the ionic positions, $n_{c}^{(A)}(r)$ and $n_{c}^{(B)}(r)$ are the core electronic densities, and $n_{v}^{(A)}(r)$ and
$n_{v}^{(B)}(r)$ are the valence electronic densities (screening clouds) associated with the $A$ - and $B$-type ions, respectively. The main aim of the NPA method is the computation of $n_{v}^{(A)}(r)$ and $n_{v}^{(B)}(r)$; it proceeds as follows.

Each $n_{v}^{(\alpha)}(r)(\alpha=A, B)$ is decomposed into two contributions, denoted $n_{v}^{\prime(\alpha)}(r)$ and $n_{v}^{\prime \prime(\alpha)}(r)$, that is, $n_{v}^{(\alpha)}(r)$ $=n_{v}^{\prime(\alpha)}(r)+n_{v}^{\prime \prime(\alpha)}(r)$. The first contribution, namely, $n_{v}^{\prime(\alpha)}(r)$, arises when an $\alpha$-type metallic ion is introduced into a jellium in which a cavity has been made, so it represents the valence electronic density induced by an external potential $V_{\text {ion }}^{\prime(\alpha)}(r)$, defined as

$$
V_{\mathrm{ion}}^{\prime(\alpha)}(r)=V_{\mathrm{ion}}^{(\alpha)}(r)-\left[-\frac{1}{r} * \nu^{(\alpha)}(r)\right],
$$

where $*$ denotes the convolution operation, $V_{\text {ion }}^{(\alpha)}(r)$ stands for the bare ionic potential, and $\nu^{(\alpha)}(r)$ is a spherical cavity screening function which integrates to the corresponding ionic valence $Z_{v}^{\alpha}$ and is chosen in order to make $V_{\text {ion }}^{\prime(\alpha)}(r)$ as weak as possible. Now, each "auxiliary ionic potential", $V_{\text {ion }}^{\prime(\alpha)}(r)$, when introduced into the uniform electron gas $\bar{n}$, induces screening valence electronic densities $n_{v}^{\prime(\alpha)}(r)$. Moreover, the contribution of the corresponding core electrons to the auxiliary ionic potential is influenced by the presence of the valence electrons; consequently $V_{\text {ion }}^{\prime(\alpha)}$ and $n_{v}^{\prime(\alpha)}(r)$ must be evaluated self-consistently. This is performed by using the DFT, by solving the Kohn-Sham equations [13], and, where the electronic exchange and correlation effects have been included, via the local-density approximation (LDA). In this way both $n_{v}^{\prime(A)}(r)$ and $n_{v}^{\prime(B)}(r)$ are obtained.

The second contribution to the induced valence electronic densities, namely, $n_{v}^{\prime \prime(\alpha)}(r)$, comes from the electronic valence density which screens, in LRT, the charge distribution associated with the cavity screening function $\nu^{(\alpha)}(r)$, that is

$$
\tilde{n}_{v}^{\prime \prime(\alpha)}(q)=-\frac{4 \pi}{q^{2}} \chi(q) \widetilde{\nu}^{(\alpha)}(q),
$$

where the tilde denotes the Fourier transform, and $\chi(q)$ is the density response function. In order to be consistent with the previous step, the exchange and correlation effects in $\chi(q)$ have been included via a LDA local field.

Now, we turn to the calculation of the local pseudopotentials $\tilde{v}_{\mathrm{ps}}^{(A)}(q)$ and $\tilde{v}_{\mathrm{ps}}^{(B)}(q)$, that within LRT reproduce the nonlinear screening charges determined by the NPA method. This is achieved by first pseudizing both $n_{v}^{(A)}(r)$ and $n_{v}^{(B)}(r)$, so as to eliminate the core orthogonality oscillations, leading to the respective induced valence electron pseudodensities $n_{\mathrm{ps}}^{(A)}(r)$ and $n_{\mathrm{ps}}^{(B)}(r)$, from which the corresponding pseudopotentials are obtained via

$$
\tilde{n}_{\mathrm{ps}}^{(A)}(q)=\chi(q) \tilde{v}_{\mathrm{ps}}^{(A)}(q), \quad \tilde{n}_{\mathrm{ps}}^{(B)}(q)=\chi(q) \tilde{v}_{\mathrm{ps}}^{(B)}(q)
$$

Finally, application of standard second-order peudopotential perturbation theory leads to the effective interatomic pair potentials, $\varphi_{\alpha \beta}(r)$, given by $(\alpha, \beta=A, B)$ 


$$
\varphi_{\alpha \beta}(r)=\frac{Z_{v}^{\alpha} Z_{v}^{\beta}}{r}+\frac{1}{(2 \pi)^{3}} \int d \vec{q} \chi(q) \tilde{v}_{\mathrm{ps}}^{(\alpha)}(q) \tilde{v}_{\mathrm{ps}}^{(\beta)}(q) e^{i \vec{q} \cdot \vec{r}}
$$

This completes the specification of the NPA method as we have applied it to compute the screening valence electronic densities and the interatomic pair potentials of a simple liquid metallic alloy $A_{x} B_{1-x}$.

\section{B. Liquid state theory}

Most of the integral-equation theories of liquids stem from the Ornstein-Zernike equation, which, for a homogeneous isotropic binary system, reads $(i, j=1,2)$

$$
h_{i j}(r)=c_{i j}(r)+\sum_{l=1}^{2} \rho_{l} h_{i l}(r) * c_{l j}(r)
$$

which defines the direct correlation functions $c_{i j}(r)$ in terms of the total correlation functions $h_{i j}(r)=g_{i j}(r)-1$, where $g_{i j}(r)$ denotes the pair distribution functions, and $\rho_{l}$ denotes the partial ionic number densities. Now, Eq. (6) is supplemented by the exact closure relation

$$
c_{i j}(r)=h_{i j}(r)-\ln \left[g_{i j}(r) e^{\beta \varphi_{i j}(r)+B_{i j}(r)}\right],
$$

where $\beta=\left(k_{B} T\right)^{-1}$ is the inverse temperature times the Boltzmann constant, $\varphi_{i j}(r)$ are the interatomic pair potentials, and $B_{i j}(r)$ denote the so-called bridge functions, for which some approximation must be made. In this work we have used the MHNC approximation under the scheme proposed in Refs. [14] and [15] (called the MHNC-REP approximation). According to this scheme, the $B_{i j}(r)$ appearing in Eq. (7) are replaced by those obtained by solving the Percus-Yevick approximation for a reference system which is chosen to be the system under study, although interacting through the repulsive part of the interatomic pair potentials only. The MHNC-REP approximation has been applied to calculate the structural properties of the liquid $\mathrm{NaK}$ [15], $\mathrm{LiNa}$ [16], and $\mathrm{NaCs}$ [17], leading to results in excellent agreement with the corresponding MD simulations.

The numerical solution of Eqs. (6) and (7) gives the partial pair distribution functions, $g_{i j}(r)$, from which both the Ashcroft-Langreth (AL) partial static structure factors [18] $S_{i j}(q)$ and the total neutron weighted static structure factor $S(q)$ are readily obtained by

$$
\begin{gathered}
S_{i j}(q)=\delta_{i j}+4 \pi \rho\left(x_{i} x_{j}\right)^{1 / 2} \int_{0}^{\infty} r^{2} h_{i j}(r) \frac{\sin q r}{q r} d r, \\
S(q)=\frac{b_{1}^{2} x_{1} S_{11}(q)+b_{2}^{2} x_{2} S_{22}(q)+2 b_{1} b_{2}\left(x_{1} x_{2}\right)^{1 / 2} S_{12}(q)}{x_{1} b_{1}^{2}+x_{2} b_{2}^{2}}
\end{gathered}
$$

where $b_{1}$ and $b_{2}$ denote the neutron scattering lengths of components 1 and 2, respectively [19]. For the discussion concerning the existence of possible ordering tendencies in a liquid binary alloy, the Bathia-Thornton concentrationconcentration partial structure factor [20] is ideally suited; this is defined as

$$
S_{\mathrm{CC}}(q)=x_{1} x_{2}\left[x_{2} S_{11}(q)+x_{1} S_{22}(q)-2\left(x_{1} x_{2}\right)^{1 / 2} S_{12}(q)\right]
$$

which in the case of the so-called "zero alloy" composition, i.e., when $x_{1} b_{1}+x_{2} b_{2}=0$, leads to $S_{\mathrm{CC}}(q)=x_{1} x_{2} S(q)$, and therefore, it can be directly probed by a single neutron scattering experiment. Closely connected to the $S_{\mathrm{CC}}(q)$ is another magnitude, the radial concentration correlation function, $\rho_{\mathrm{CC}}(r)$, which was introduced by Ruppersberg and Egger [21] to analyze the ordering tendencies in disordered binary systems; this is defined as

$$
\rho_{\mathrm{CC}}(r)=\frac{2 r}{\pi} \int_{0}^{\infty} q\left[\frac{S_{\mathrm{CC}}(q)}{x_{1} x_{2}}-1\right] \sin (q r) d r
$$

and it can also be expressed in terms of the partial pair distribution functions $g_{i j}(r)$ as

$$
\rho_{\mathrm{CC}}(r)=4 \pi r^{2} \rho x_{1} x_{2}\left[g_{11}(r)+g_{22}(r)-2 g_{12}(r)\right],
$$

and becomes negative (positive) for distances with preferred heterocoordination (homocoordination).

\section{MOLECULAR DYNAMICS SIMULATIONS}

The present study has been completed by performing MD simulations for both liquid alloys. The simulations have been carried out by using the NPA-based interatomic pair potentials, and for both alloys we have just focused on their respective zero alloy compositions, i.e., $\mathrm{Li}_{0.61} \mathrm{Na}_{0.39}$ and $\mathrm{Li}_{0.7} \mathrm{Mg}_{0.3}$. In this way, we have obtained their corresponding static structure as well as some dynamic properties.

In the simulations, we considered a cubic box with 600 particles and periodic boundary conditions. After an equilibration period of 10000 time steps, we computed the properties during a run of 20000 configurations. The time step was $2 \mathrm{fs}$, and the leap-frog Verlet algorithm with coupling to a thermal bath as proposed by Berendsen et al. [22] was used. The partial radial distribution functions $g_{i j}(r)$, the velocity autocorrelation functions $C_{i}(t)$ and the mean square displacements were computed during the simulations, whereas the partial static structure factors $S_{i j}(q)$ were calculated by Fourier transforming the obtained $g_{i j}(r)$ 's [23]. The finite size of the simulation box, however, imposes a limit in the wavelength of fluctuations, and therefore the results for the structure factors are reliable for $q>q_{\min }$, where $q_{\min }$ is in all cases around $0.24 \AA^{-1}$.

\section{RESULTS: Li-Na SYSTEM}

The Li-Na liquid alloy has attracted much attention because it is a typical phase separating system. The phase diagram is dominated by a region of two immiscible liquids, and the phase separation curve, which has been experimentally determined by means of density and resistivity measurements [24-26], shows a consolute point located at $T$ $\approx 577 \mathrm{~K}$ and composition $x_{\mathrm{Li}} \approx 0.64$; this is close to the zero alloy composition $\left(x_{\mathrm{Li}} \approx 0.61\right)$ for which the total static structure factor $S(q)$ reduces to the concentration-concentration partial structure factor, namely, $S(q)=S_{\mathrm{CC}}(q) / x_{\mathrm{Li}} x_{\mathrm{Na}}$, because of the negative scattering length of the ${ }^{7} \mathrm{Li}$ isotope. For the zero alloy composition, Ruppersberg and Knoll [5] mea- 
sured the total $S(q)$ at several temperatures within the range $590-725 \mathrm{~K}$. The obtained experimental $S(q)$ 's are dominated by a strong small-angle scattering, which is a typical characteristic of a phase separating system, and the oscillations of the $S(q)$ 's beyond the small-angle region are extremely small, with a characteristic ripple around $q$ $\approx 2.2 \AA^{-1}$.

Several theoretical works, using either semiempirical [27-30] or more fundamental [16,31-34] approaches, have already been attempted in order to calculate the static structure and/or some thermodynamic magnitudes of this alloy. Among those based on semiempirical models, we mention Refs. [28-30], which basically focused on the zero alloy composition at $T=595 \mathrm{~K}$, and where the interatomic pair potentials in the alloy were modeled by hard sphere Yukawa potentials. The parameters involved in the model were obtained from a global fitting of the experimental $S_{\mathrm{CC}}(q)$, and the static structure of the alloy was computed by resorting to the mean spherical approximation theory of liquids. Although use was made of several fitted parameters, these works gave some insight into the interatomic interactions.

On the other hand, studies based on more fundamental approaches used pseudopotential perturbation theory combined with either perturbative or integral equation theories of liquids. For example, Stroud and co-workers [31,32] calculated the spinoidal line of the $\mathrm{Li}_{x} \mathrm{Na}_{1-x}$ alloy, using the empty-core model (ECM) pseudopotential [35] to derive interatomic pair potentials, and a perturbative approach to obtain the static structure. Although they just obtained a qualitative agreement with the experimental spinoidal line, it must be stressed that no parameters were included in the model.

Also, Hoshino and co-workers [33,34] studied the $\mathrm{Li}_{0.61} \mathrm{Na}_{0.39}$ alloy by using either the ECM [33] or the Hoshino-Young pseudopotential [34] for $\mathrm{Li}$, whereas the ECM was used for Na. Their results for the static structure, evaluated by the HNC theory [33] and MD simulations [34], qualitatively reproduced the diverging behavior of $S(q$ $\rightarrow 0$ ), but did not show the ripple at $q \approx 2.2 \AA^{-1}$. More recently, Mori, Hoshino, and Watabe [16] studied the static structure of the $\mathrm{Li}_{x} \mathrm{Na}_{1-x}$ alloy by combining a nonlocal pseudopotential [36] for $\mathrm{Li}$ with the Hasegawa et al. [37] pseudopotential for $\mathrm{Na}$, whereas the static structural properties were calculated by both MD simulations and the MHNC-REP theory of liquids. Although their results reproduced the ripple at $q \approx 2.2 \AA^{-1}$, the diverging behavior of $S(q \rightarrow 0)$ was now grossly underestimated by around a factor of 50 .

Therefore, it may be concluded that an accurate structural description must start from a derivation of adequate interatomic interactions for the alloy. In this section we use the NPA method to obtain the effective interatomic pair potentials in the alloy. Specifically, we study the structural features of liquid $\mathrm{Li}_{x} \mathrm{Na}_{1-x}$ at $T=725 \mathrm{~K}$ and several concentrations; moreover for the particular case of the zero alloy composition, i.e., $\mathrm{Li}_{0.61} \mathrm{Na}_{0.39}$, we complete the study by performing MD simulations for several temperatures within the range $540 \mathrm{~K} \leqslant T \leqslant 725 \mathrm{~K}$. The total ionic number densities used in the calculations have been taken from the experimental results of Jost et al. [38], and Table I shows the specific thermodynamic states for which the present study has been carried out.
TABLE I. Thermodynamic states of $\mathrm{Li}_{x} \mathrm{Na}_{1-x}$ studied in this work. $\rho$ is the total ionic number density taken from Ref. [38].

\begin{tabular}{lccccc}
\hline \hline$x=x_{\mathrm{Li}}$ & $\begin{array}{c}T=725 \\
\rho\left(\AA^{-3}\right)\end{array}$ & 690 & 640 & 590 & 540 \\
\hline 0.0 & 0.022040 & & & & \\
0.40 & 0.027237 & & & & \\
0.61 & 0.031085 & 0.03136 & 0.03171 & 0.03218 & 0.03258 \\
0.80 & 0.035640 & & & & \\
1.0 & 0.042141 & & & & \\
\hline \hline
\end{tabular}

\section{A. Interatomic pair potentials}

Figure 1 shows the NPA interatomic pair potentials obtained for three different concentrations of the alloy $\mathrm{Li}_{x} \mathrm{Na}_{1-x}$ at $T=725 \mathrm{~K}$. First, an interesting feature of the present NPA pair potentials is that $\varphi_{\mathrm{LiNa}}(r)$ is, for all concentrations, weaker than the average of $\varphi_{\mathrm{LiLi}}(r)$ and $\varphi_{\mathrm{NaNa}}(r)$. As the structural properties of the alloy are closely linked to the relative magnitudes of the $\varphi_{i j}(r)$, this feature will have important consequences in the alloy which will be analyzed in Sec. IV B.

It is observed that when the alloy concentration is varied, going from pure $\mathrm{Na}$ to pure $\mathrm{Li}$, all the three pair potentials become shallower. This behavior can be understood in terms of the changes in the electronic screening as follows. According to Eq. (5), the effective interatomic pair potential has a direct Coulombic repulsive term and an attractive, electron mediated, contribution. The latter is screened by the presence of other electrons. As the concentration of $\mathrm{Li}$ is increased, the mean electron density in the alloy also increases, and therefore the screening of the attractive part of the pair potentials is more effective, leading finally to less attractive (shallower) potentials. Moreover, it is also observed that the changes undergone by the pair potentials are somewhat more marked for $\varphi_{\mathrm{LiLi}}(r)$, which is the deepest one.

\section{B. Structural properties}

The calculation of the liquid structure at several concentrations has been carried out by combining the NPA interatomic pair potentials, derived in Sec. IV A, with the MHNC-REP theory of liquids. First, the calculated partial pair distribution functions $g_{i j}(r)$ are shown in Fig. 1. Furthermore, in Fig. 2 we compare the theoretical $g_{i j}(r)$ with the MD ones for $\mathrm{Li}_{0.61} \mathrm{Na}_{0.39}$ at $T=725 \mathrm{~K}$. The agreement is

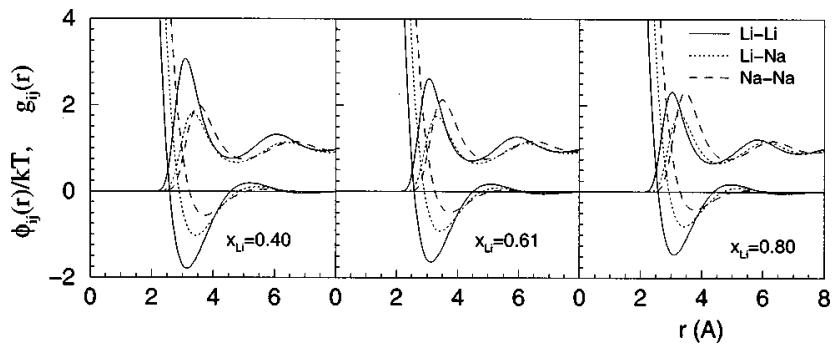

FIG. 1. NPA interatomic pair potentials $\varphi_{i j}(r)$ and partial pair distribution functions $g_{i j}(r)$ for the liquid $\mathrm{Li}_{0.4} \mathrm{Na}_{0.6}, \mathrm{Li}_{0.61} \mathrm{Na}_{0.39}$, and $\mathrm{Li}_{0.8} \mathrm{Na}_{0.2}$ alloys at $T=725 \mathrm{~K}$. 


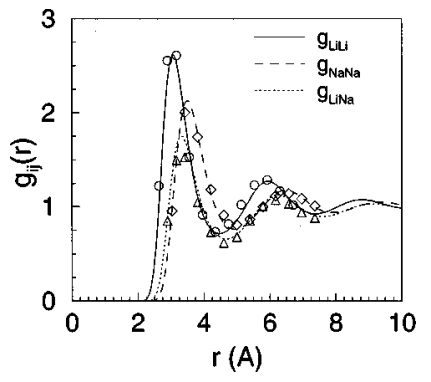

FIG. 2. Comparison, for $\mathrm{Li}_{0.61} \mathrm{Na}_{0.39}$ at $T=725 \mathrm{~K}$, between the partial pair distribution functions $g_{i j}(r)$ obtained by the MHNCREP theory (lines) and those obtained by molecular dynamics simulations (symbols).

excellent, showing once more the reliability of the MHNCREP theory, which was already observed by Mori and coworkers [15-17].

According to the previously obtained tendencies for the $\varphi_{i j}(r)$, it is observed that $g_{\mathrm{LiLi}}(r)$ undergoes substantial changes, whereas both $g_{\mathrm{LiNa}}(r)$ and $g_{\mathrm{NaNa}}(r)$ change slightly. In fact, Fig. 1 shows that when moving from pure $\mathrm{Na}$ to pure $\mathrm{Li}$, the height of the main peak of $g_{\mathrm{LiLi}}(r)$ decreases, whereas the height of $g_{\mathrm{LiNa}}(r)$ practically remains constant and that of $g_{\mathrm{NaNa}}(r)$ increases slightly. An interesting feature to be observed in Fig. 1 is that, for all concentrations, $g_{\mathrm{LiNa}}(r)$ remains smaller than both $g_{\mathrm{LiLi}}(r)$ and $g_{\mathrm{NaNa}}(r)$; therefore, the global configuration predicted by the theoretical $g_{i j}(r)$ is a tendency toward phase separation. A simple way of characterizing this segregating tendency is by means of the Warren-Cowley [39-41] short range order (SRO) parameter for the first neighbor shell, $\alpha_{1}^{(i)}$, which is defined as

$$
\alpha_{1}^{(i)}=1-\frac{n_{i j}}{x_{j}\left(n_{i i}+n_{i j}\right)}, \quad j \neq i=1,2,
$$

where $x_{j}$ is the concentration of the $j$-type particles, and $n_{i j}$ is the number, within a spherical volume of radius $R_{i j}$, of $j$-type particles around an $i$-type particle. $n_{i j}$ can be calculated from the partial pair distribution functions $g_{i j}(r)$ by

$$
n_{i j}=4 \pi \rho x_{j} \int_{0}^{R_{i j}} r^{2} g_{i j}(r) d r
$$

where $R_{i j}$ has been identified as the position of the first minimum of the corresponding $g_{i j}(r)$. We note that although other prescriptions could be used for the evaluation of $n_{i j}$, leading to slightly different results [42], this is not really important, as we are interested in the trends exhibited by $\alpha_{1}^{(i)}$ when the alloy concentration or the temperature are changed.

$\alpha_{1}^{(i)}$ provides insight into the local arrangement of particles in the mixture. For a random distribution of atoms, $\alpha_{1}^{(i)}=0$, whereas a positive (negative) value for $\alpha_{1}^{(i)}$ suggests a segregating (heterocoordinating) tendency. We have evaluated both $\alpha_{1}^{(\mathrm{Li})}$ and $\alpha_{1}^{(\mathrm{Na})}$ for the liquid $\mathrm{Li}_{x} \mathrm{Na}_{1-x}$ alloy at $T$ $=725 \mathrm{~K}$; the obtained results are shown in Table II, and clearly confirm the segregating tendency. However, our theoretical calculations predict that the segregating tendency is more marked at $x_{\mathrm{Li}} \approx 0.5$, whereas the experiment shows that it should be at $x_{\mathrm{Li}} \approx 0.64$. Moreover, Table II shows that for
TABLE II. Calculated values of coordination numbers $n_{i j}$ and the Warren-Cowley SRO parameters $\alpha_{1}^{(i)}$ for the $\mathrm{Li}_{x} \mathrm{Na}_{1-x}$ alloy at $T=725 \mathrm{~K}$.

\begin{tabular}{lrccrcl}
\hline \hline$x_{\mathrm{Li}}$ & $n_{\mathrm{LiLi}}$ & $n_{\mathrm{LiNa}}$ & $n_{\mathrm{NaNa}}$ & $n_{\mathrm{NaLi}}$ & $\alpha_{1}^{(\mathrm{Li})}$ & $\alpha_{1}^{(\mathrm{Na})}$ \\
\hline 0.4 & 5.8 & 6.7 & 8.5 & 4.5 & 0.107 & 0.140 \\
0.61 & 8.0 & 4.6 & 6.3 & 7.1 & 0.064 & 0.131 \\
0.8 & 10.3 & 2.5 & 3.7 & 10.2 & 0.020 & 0.083 \\
\hline \hline
\end{tabular}

$x_{\mathrm{Li}}=0.61$, the $\mathrm{Li}$ atoms have eight $\mathrm{Li}$ atoms as nearest neighbors, which is somewhat higher than the value of seven $\mathrm{Li}$ atoms suggested by Ruppersberg and Knoll [5], based on the $\rho_{\mathrm{CC}}(r)$ obtained from their experimental $S(q)$. On the other hand, Fig. 2 shows that the preferred Li-Li and Na-Na distances are around 3 and $3.5 \AA$, respectively, in agreement with the values derived by the same authors.

As mentioned above, the static structure factors of liquid $\mathrm{Li}_{x} \mathrm{Na}_{1-x}$ at $T=590 \mathrm{~K}$ and $x_{\mathrm{Li}}=0.40,0.61$, and 0.80 , as well as for $T=725 \mathrm{~K}$ and $x_{\mathrm{Li}}=0.61$, were measured by Ruppersberg and Knoll [5] using neutron diffraction techniques. For this concentration and temperature range, the experimental static structure factors $S_{\text {exp }}(q)$ 's show some common features: (i) A rapid increase of $S_{\text {exp }}(q)$ as $q \rightarrow 0$. (ii) The oscillations of $S_{\exp }(q)$ beyond $q \geqslant 2.0 \AA^{-1}$ are extremely weak, which is characteristic of alloys with weak chemical ordering. (iii) For the zero alloy composition $\mathrm{Li}_{0 . .61} \mathrm{Na}_{0.39}$, there appears a characteristic ripple with two peaks at $q \approx 2.0$ and $q \approx 2.5 \AA^{-1}$, respectively.

Figure 3 shows a comparison for $\mathrm{Li}_{0.61} \mathrm{Na}_{0.39}$ at $T$ $=590 \mathrm{~K}$, between $S_{\text {exp }}(q)$ and the MD-based $S_{\mathrm{MD}}(q)$. It is observed that $S_{\mathrm{MD}}(q)$ can successfully reproduce the main features of the experimental one, especially the quick increase of $S_{\text {exp }}(q \rightarrow 0)$ and the two peaks at $q \approx 2$ and $2.5 \AA^{-1}$. Moreover, by extrapolating the small- $q$ results we obtain $S_{\mathrm{MD}}(q=0) \approx 135$, which is close to the value $S_{\exp }(q$ $=0) \approx 110$, extrapolated by Ruppersberg and Knoll from their experimental results. Concerning the two peaks at $q$ $\approx 2$ and $2.5 \AA^{-1}$, their origin can be traced back to the shape of the AL partial static structure factors $S_{i j}(q)$, which are shown in the inset of Fig. 3. $S_{\mathrm{NaNa}}(q)$ shows a peak at $q \approx 2 \AA^{-1}$, the peak of $S_{\mathrm{LiLi}}(q)$ is at $q \approx 2.5 \AA^{-1}$, and the peak of $S_{\mathrm{LiNa}}(q)$ is at $q \approx 2.25 \AA^{-1}$. Taking into account the expression for $S(q)$ in Eq. (9), and that the neutron scattering

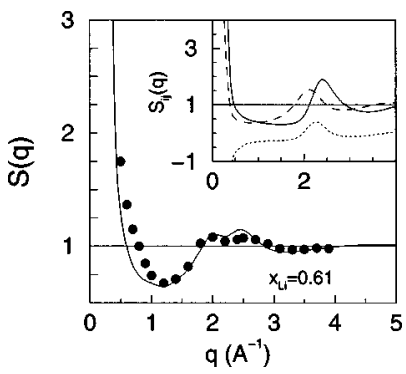

FIG. 3. Total static structure factor $S(q)$ for the $\mathrm{Li}_{0.61} \mathrm{Na}_{0.39}$ liquid alloy at $T=590 \mathrm{~K}$. Solid line: molecular dynamics results. Full circles: experimental data [5]. The inset shows the corresponding molecular dynamics Ashcroft-Langreth partial static structure factors: $S_{\mathrm{LiLi}}(q)$ (solid line), $S_{\mathrm{NaNa}}(q)$ (dashed line), and $S_{\mathrm{LiNa}}(q)$ (dotted line). 


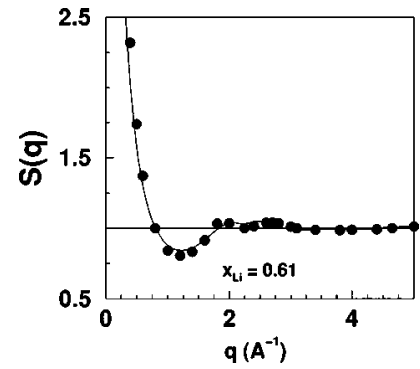

FIG. 4. Comparison between the theoretical (solid line) and the experimental [5] (full circles) total static structure factor $S(q)$ for the $\mathrm{Li}_{0.61} \mathrm{Na}_{0.39}$ liquid alloy at $T=725 \mathrm{~K}$.

length of ${ }^{7} \mathrm{Li}$ is negative whereas that of $\mathrm{Na}$ is positive, then it is clear that the two peaks in $S_{\text {exp }}(q)$ originate from the difference in the peak positions of $S_{i j}(q)$. Obviously, the ultimate reason for the peak positions of $S_{i j}(q)$ relies on the shape of the NPA interatomic pair potentials.

We emphasize that, unlike previous works using MD simulation techniques combined with other interatomic pair potentials [16,34], to our knowledge this is the first work in which both features of $S_{\text {exp }}(q)$ are quantitatively reproduced simultaneously, and this therefore provides further confidence in the NPA-based interatomic pair potentials.

On the other hand, a full comparison theory/experiment can only be carried out at the zero alloy composition and $T$ $=725 \mathrm{~K}$. Although the NPA method can provide interatomic pair potentials for $T=590 \mathrm{~K}$, the fact that $S(q)$ increases to very high values for small wave vectors produces numerical problems in the iterative solution of Eqs. (6) and (7). Therefore, we have computed the static structure for the higher temperature $T=725 \mathrm{~K}$ for which, although $S(q)$ still takes substantial values for small- $q$ values, it is nevertheless possible to attain a numerical solution for the liquid state theory.

In Fig. 4, we show a comparison between the theoretical and experimental total static structure factor for $\mathrm{Li}_{0.61} \mathrm{Na}_{0.39}$ at $T=725 \mathrm{~K}$. The theory reproduces rather well the main features of $S_{\exp }(q)$; moreover, $S_{\mathrm{th}}(q=0) \approx 5$, whereas the extrapolated [5] $S_{\text {exp }}(q=0) \approx 7$. The theoretical results obtained for other concentrations at $725 \mathrm{~K}$ are shown in Fig. 5, where they are compared with the measured $S(q)$ at $590 \mathrm{~K}$. It is observed that the main features present in the experimental structure factor, which also persist at the higher temperature [5], are well reproduced by the theoretical approach.

As already pointed out, we have also performed MD simulations for the zero alloy composition at several temperatures within the range $540 \mathrm{~K} \leqslant T \leqslant 725 \mathrm{~K}$. First, from the obtained partial pair distribution functions $g_{i j}(r)$, the following conclusions are obtained for the static structure at the zero alloy composition. When the temperature is varied within the range $540 \mathrm{~K} \leqslant T \leqslant 725 \mathrm{~K}$, we obtain the following: (i) The nearest-neighbor distances for $\mathrm{Li}-\mathrm{Li}, \mathrm{Li}-\mathrm{Na}$, and $\mathrm{Na}-\mathrm{Na}$ remain practically constant at around $3,3.3$, and 3.5 $\AA$, respectively. (ii) The heights of the main peaks of $g_{\mathrm{LiLi}}(r)$ and $g_{\mathrm{NaNa}}(r)$ increase by $25 \%$ and $16 \%$, respectively whereas that of $g_{\mathrm{LiNa}}(r)$ decreases by $25 \%$, as the temperature is decreased. (iii) For a $\mathrm{Li}$ ion, the total average number of nearest neighbors (NN's) slightly changes from 12.6 (for $T=725 \mathrm{~K}$ ) to 13.1 (for $T=540 \mathrm{~K}$ ); however, the average

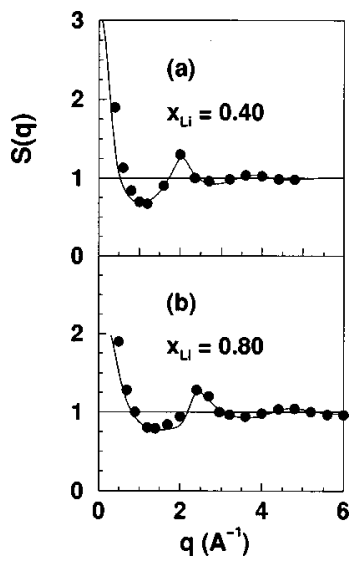

FIG. 5. (a) Theoretical total static structure factor $S(q)$ for the $\mathrm{Li}_{0.4} \mathrm{Na}_{0.6}$ liquid alloy at $T=725 \mathrm{~K}$ (solid line). For comparison, the full circles show the experimental result [5] at $T=590 \mathrm{~K}$. (b) Same as before, but for $\mathrm{Li}_{0.8} \mathrm{Na}_{0.2}$.

number of its Li-type NN's changes from 8.0 to 9.5 whereas the average number of its Na-type NN's decreases from 4.6 to 3.6. Similarly, for a $\mathrm{Na}$ ion, the total average number of NN's practically remains constant at 13.4 ; however, the average number of its Na-type NN's increases from 6.2 to 7.8 , whereas that of its Li-type NN's decreases from 7.1 to 5.6. (iv) By resorting to the Warren-Cowley SRO parameter, we obtain that $\alpha_{1}^{(\mathrm{Li})}$ varies from 0.064 (for $T=725 \mathrm{~K}$ ) to 0.30 (for $T=540 \mathrm{~K}$ ), whereas the corresponding variation for the $\alpha_{1}^{(\mathrm{Na})}$ ranges from 0.131 to 0.314 . In summary, the above results clearly indicate the existence of a segregating behavior which becomes more marked as the temperature is decreased.

Now, by using the obtained MD static structural functions, namely, $g_{i j}(r)$, we have quantitatively investigated the phase separation region. This has been carried out by means of the local mole fractions method [43-47]. For a binary system, the local mole fractions $x_{i i}$ are defined as

$$
x_{i i}=\frac{n_{i i}}{n_{i i}+n_{i j}}, \quad j \neq i=1,2
$$

where $n_{i j}$ have already been defined in connection with Eq. (14). Although some discussion $[43,48-50]$ has been devoted to the identification of a suitable upper integration limit $R_{i j}$ in Eq. (14), we have taken the same $R_{i j}$ as used in the evaluation of the Warren-Cowley SRO parameter. The important magnitude in this method is $x_{s}-1=x_{11}+x_{22}-1$, which provides information about homogeneous or inhomogeneous regions of states in the mixture. For a homogeneous phase, when both particle species are nearly equally distributed, $x_{s}-1$ is a small number; however, for a demixed state, particles of equal species predominate the distribution and therefore $x_{s}-1$ grows, becoming a relatively large number. For more details we refer the reader to Refs. [44,45]. Figure 6 shows a plot of $x_{s}-1$ as a function of the temperature for the zero alloy composition $\mathrm{Li}_{0.61} \mathrm{Na}_{0.39}$. The pronounced increase in the slope of $x_{s}-1$ suggests a phase transition to the demixing state, which, according to the obtained MD-based results, takes place at a temperature somewhat smaller than $640 \mathrm{~K}$. 


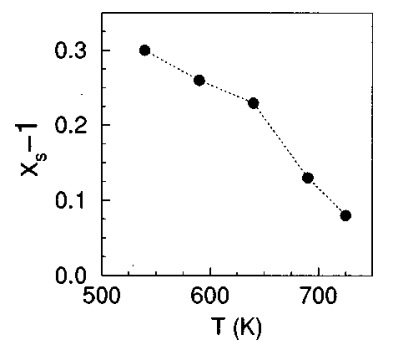

FIG. 6. Sum of the local mole fractions minus 1 for the "zero alloy' composition $\mathrm{Li}_{0.61} \mathrm{Na}_{0.39}$ at different temperatures.

\section{Dynamic properties}

By means of MD simulations, we have also evaluated some time dependent properties for the $\mathrm{Li}_{0.61} \mathrm{Na}_{0.39}$ liquid alloy at several temperatures. Specifically, we have calculated the velocity autocorrelation functions $C_{i}(t)$, the mean square displacements, and the self-diffusion coefficients $D_{i}$ associated with the screened $\mathrm{Li}$ and $\mathrm{Na}$ ions in the alloy. First, in Fig. 7 we show, for several temperatures, the obtained results for $C_{\mathrm{Li}}(t)$ and $C_{\mathrm{Na}}(t)$. It is observed that, for all temperatures, the minima exhibited by $C_{\mathrm{Li}}(t)$ (backscattering effects) are always deeper and appear at shorter times than those for the corresponding $C_{\mathrm{Na}}(t)$. This can be associated with the smaller mass of the $\mathrm{Li}$ ion, since the backscattering effects measure the tendency for a reversal of the initial velocity of a tagged atom due to collisions with their neighbors [51].

Concerning the self-diffusion coefficients $D_{i}$, its evaluation has been carried out from both the time integrals of the corresponding $C_{i}(t)$ functions and also from the slopes of the respective mean square displacements [52]; the results from the two methods are in good agreement, and are summarized in Table III. First, we note that, to our knowledge, there are no experimental results for the self-diffusion coefficients in this alloy system. The present results show that, within the studied temperature range, both self-diffusion coefficients are rather similar, and, moreover, that the obtained values corresponding to the ions with the smaller mass and size (Li) are slightly lower than those for the larger ions $(\mathrm{Na})$. This behavior, which departs largely from the simple

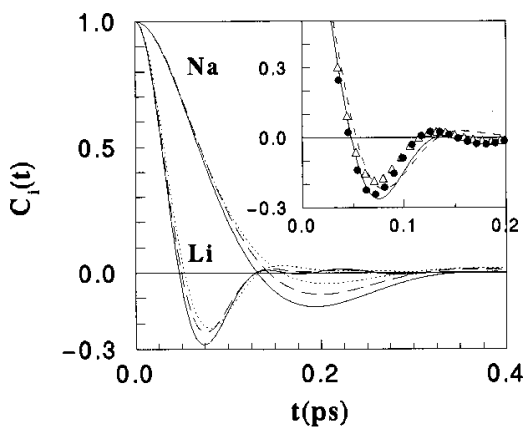

FIG. 7. Molecular dynamics normalized velocity autocorrelation functions $C_{\mathrm{Li}}(t)$ and $C_{\mathrm{Na}}(t)$, for the $\mathrm{Li}_{0.61} \mathrm{Na}_{0.39}$ liquid alloy at different temperatures. Solid line: $T=540 \mathrm{~K}$. Dashed line: $T$ $=640 \mathrm{~K}$. Dotted line: $T=725 \mathrm{~K}$. The inset shows the MD results for the $C_{\mathrm{Li}}(t)$ in the alloy at $T=590 \mathrm{~K}$ (full line) and $725 \mathrm{~K}$ (dashed line) and the same results for pure $\mathrm{Li}$ at $T=590 \mathrm{~K}$ (full circles) and $725 \mathrm{~K}$ (open triangles).
TABLE III. Diffusion coefficients (in $10^{-5} \mathrm{~cm}^{2} / \mathrm{s}$ ) for the $\mathrm{Li}$ and $\mathrm{Na}$ ions in the liquid $\mathrm{Li}_{0.61} \mathrm{Na}_{0.39}$ alloy at several temperatures.

\begin{tabular}{rrrrrc}
\hline \hline & $540 \mathrm{~K}$ & $590 \mathrm{~K}$ & $640 \mathrm{~K}$ & $690 \mathrm{~K}$ & $725 \mathrm{~K}$ \\
\hline $\mathrm{Li}$ & 9.7 & 11.8 & 14.6 & 17.6 & 19.3 \\
$\mathrm{Na}$ & 10.4 & 12.8 & 15.2 & 17.6 & 19.3 \\
\hline \hline
\end{tabular}

scaling rule $D_{\mathrm{Li}} / D_{\mathrm{Na}}=\sqrt{m_{\mathrm{Na}} / m_{\mathrm{Li}}} \approx 1.8$ as derived from the thermal velocities only, clearly states the important role played by the interatomic pair potentials in the diffusion process. On the other hand, Casas, González, and González [53] recently performed MD simulations for liquid $\mathrm{Li}$ at several thermodynamic states along the coexistence line, by using NPA-based interatomic pair potentials. In Fig. 7, we plot the obtained $C_{\mathrm{Li}}(t)$ for pure $\mathrm{Li}$ at two thermodynamic states, namely, $T=590 \mathrm{~K}, \rho=0.0435 \AA^{-3}$, and $T=725 \mathrm{~K}, \rho$ $=0.04214 \AA^{-3}$. For both temperatures, it is observed that the $C_{\mathrm{Li}}(t)$ in the alloy exhibits a somewhat deeper and wider minimum than in the pure system. Although the averaged number of NN's of a Li ion in the pure system is somewhat larger than in the alloy at the same temperature, the presence of heavier $\mathrm{Na}$ ions as $\mathrm{NN}$ in the alloy leads to an enhancement of the backscattering effects in the alloy, and therefore the corresponding $D_{\mathrm{Li}}$ is slightly smaller (around 5\%) than in the pure system. Similar, but opposite, effects take place in the case of $C_{\mathrm{Na}}(t)$, leading to a $D_{\mathrm{Na}}$ which is somewhat larger in the alloy than in the pure system.

\section{RESULTS: Li-Mg SYSTEM}

The Li-Mg phase diagram shows that $\mathrm{Li}$ and $\mathrm{Mg}$ are completely miscible in the liquid state and even show some heterocoordination. Ruppersberg et al. [4] performed a neutron diffraction study of the total static structure factor for the " 'zero alloy" composition, namely, $\mathrm{Li}_{0.7} \mathrm{Mg}_{0.3}$, at two temperatures $T=695$ and $875 \mathrm{~K}$. Their experimental results, for both temperatures, show that the total $S(q)$ are rather structureless, with $S(q \rightarrow 0)$ being smaller than 1, which suggests some preferred heterocoordination of nearest neighbors.

Up to date, few theoretical works have been devoted to the Li-Mg alloy system; most of them have focused in the solid phase. Among these we should mention the work of Ref. [54], who calculated, at several concentrations, the elastic constants and the heat of mixing for the body centered cubic solid phase, using interatomic pair potentials derived from the pseudopotentials of Ref. [55]. We note that these pseudopotentials are based on the same philosophy as the present NPA method, although their practical implementation differs from the approach followed in this work. It is also worth mentioning the work of Hafner, who studied both the solid [56] and liquid [57] phases of the Li-Mg alloy by using effective interatomic pair potentials derived from the first principles optimized pseudopotentials (OPW's) of Harrison [58]. For the solid phase, he obtained reasonable results for the enthalpies, volumes of formation, and structure and stability range of the alloy phases. However, when applied to the liquid phase, using a hard sphere reference system combined with the Gibbs-Bogolyubov variational scheme, the obtained structural results did not suggest the existence of heterocoordinating tendencies. A further study was also per- 
TABLE IV. Input data for the series $\mathrm{Li}_{x} \mathrm{Mg}_{1-x}$ at $T=887 \mathrm{~K}$, studied in this work. $\rho$ is the total ionic number density taken from Refs. [4,59].

\begin{tabular}{ll}
\hline \hline$x=x_{\mathrm{Li}}$ & $\rho\left(\AA^{-3}\right)$ \\
\hline 0.0 & 0.03967 \\
0.30 & 0.04011 \\
0.50 & 0.04041 \\
0.70 & 0.04071 \\
0.90 & 0.04102 \\
1.0 & 0.04117 \\
\hline \hline
\end{tabular}

formed in Ref. [59] by modeling the interatomic pair potentials in terms of the hard-spheres-Yukawa system, and using the mean spherical approximation theory of liquids to obtain the static structural functions. Although this approach included some fitting parameters, it had the interesting feature of providing analytical expressions for both the structural functions and some thermodynamic magnitudes. Among the several semiempirical studies performed for the liquid phase, we mention the recent work by Karaoglu and Young [60] which was just concerned with the study of the thermodynamic properties of the liquid alloy by resorting to the conformal solution model.

In this work we have calculated the static structure of $\mathrm{Li}_{x} \mathrm{Mg}_{1-x}$ at $T=887 \mathrm{~K}$ for the concentrations $x_{\mathrm{Mg}}$ $=0.1,0.3,0.5$, and 0.7. The experimental ionic number densities are taken from Refs. [4,59], and in Table IV we show the specific thermodynamic states for which the present study has been carried out. For each concentration, the corresponding interatomic pair potentials were calculated by the NPA method, and the static structure was obtained by the MHNC-REP approximation. Moreover, for the zero alloy composition at $T=887 \mathrm{~K}$, MD simulations have also been performed.

\section{A. Interatomic potentials}

Figure 8 shows the NPA interatomic pair potentials obtained for three different concentrations of the alloy $\mathrm{Li}_{x} \mathrm{Mg}_{1-x}$ at $T=887 \mathrm{~K}$. First, it is observed that $\varphi_{\mathrm{LiMg}}(r)$ is, for all concentrations, somewhat deeper than the average of $\varphi_{\mathrm{LiLi}}(r)$ and $\varphi_{\mathrm{MgMg}}(r)$; therefore a heterocoordination tendency should be expected. This will be examined in Sec. VB, where we analyze the static structural properties of the alloy.

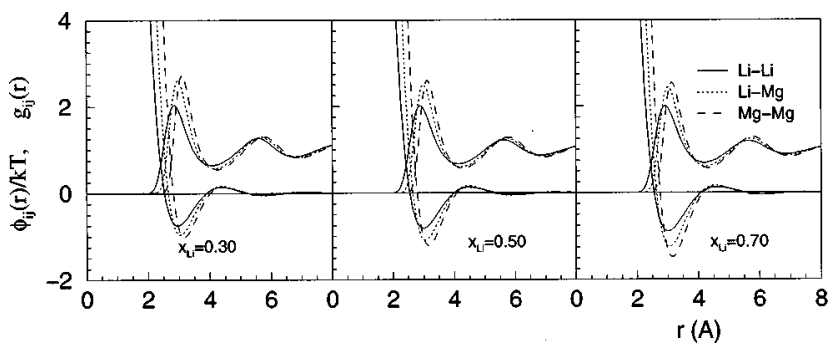

FIG. 8. NPA interatomic pair potentials $\varphi_{i j}(r)$, and partial pair distribution functions $g_{i j}(r)$, for the liquid $\mathrm{Li}_{0.3} \mathrm{Mg}_{0.7}, \mathrm{Li}_{0.5} \mathrm{Mg}_{0.5}$, and $\mathrm{Li}_{0.7} \mathrm{Mg}_{0.3}$ alloys at $T=887 \mathrm{~K}$.

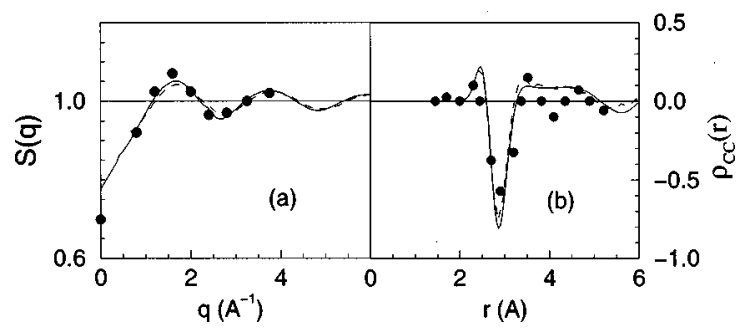

FIG. 9. (a) Theoretical total static structure factor $S(q)$ for the $\mathrm{Li}_{0.7} \mathrm{Mg}_{0.3}$ liquid alloy at $T=887 \mathrm{~K}$ (solid line). The dashed line shows the molecular dynamics results, whereas the full circles show the experimental results $[5,4]$ at $T=875 \mathrm{~K}$. (b) Radial concentration correlation function.

The changes undergone by the interatomic pair potentials as a function of the alloy concentration can again be explained in terms of the changes in the electronic screening. When the alloy concentration is varied, moving from pure $\mathrm{Mg}$ to pure $\mathrm{Li}$, the ionic number density is increased; however, conversely, the electronic density decreases as a consequence of the valence difference. Therefore the electronic screening of the attractive part of the pair potentials becomes less effective, and therefore the potentials become more attractive (deeper). We also note that the changes are more marked for $\varphi_{\mathrm{MgMg}}(r)$, which is the deepest, than for the other two potentials.

It is worth mentioning that the main features of the present effective interatomic pair potentials are shared by the OPW-based potentials derived by Hafner [57]. Although the OPW-based potentials show repulsive cores which are around $20 \%$ smaller than the present potentials, he also obtained that $\varphi_{\mathrm{MgMg}}(r)$ is the deeper one, whereas $\varphi_{\mathrm{LiMg}}(r)$ is also slightly deeper than the average of $\varphi_{\mathrm{LiLi}}(r)$ and $\varphi_{\mathrm{MgMg}}(r)$; therefore, we suspect that the failure in predicting the heterocoordinating tendencies for the liquid alloy could be due to the liquid state theory used (a hard sphere reference system within a variational approach) rather than a consequence of the effective interatomic pair potentials.

On the other hand, a comparison with the pair potentials used in Ref. [54], shows rather strong differences. In fact, the latter set of authors obtained that $\varphi_{\mathrm{LiLi}}(r)$ is the deeper potential (more than $50 \%$ deeper than $\varphi_{\mathrm{MgMg}}$ ), whereas $\varphi_{\mathrm{LiMg}}$ remains rather close to $\varphi_{\mathrm{LiLi}}$. Therefore, it leads to rather strong ordering tendencies which, according to the authors, grossly overestimate the experimental results for the solid phase.

\section{B. Structural properties}

The liquid structure has been obtained by combining the previous NPA pair potentials with the MHNC-REP theory of liquids, and in Fig. 8 we show the obtained partial pair distribution functions $g_{i j}(r)$. However, a comparison with the experimental results can be carried out at the level of the static structure factor only, and this is performed in Fig. 9 for the "zero alloy" composition $\mathrm{Li}_{0.7} \mathrm{Mg}_{0.3}$. It is observed that $S_{\mathrm{th}}(q)$ reproduces rather well the main features of $S_{\exp }(q)$ [4], although the value at $q \rightarrow 0$ is slightly overestimated. In the figure we have also included, for comparison, the corresponding MD result, which closely follows $S_{\mathrm{th}}(q)$. 


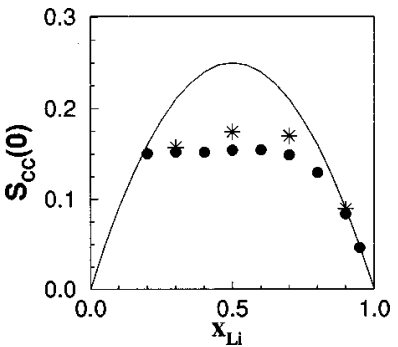

FIG. 10. Concentration dependence of $S_{\mathrm{CC}}(0)$, for the liquid Li-Mg alloy at $T=887 \mathrm{~K}$. Full circles: Experimental results from Ref. [61]. Asterisks: theoretical results. Line: ideal mixture result.

We previously mentioned that our derived NPA pair potentials suggest that some heterocoordinating tendency should be expected. Now, by using the theoretical $g_{i j}(r)$, we have evaluated the Warren-Cowley SRO parameters, and we obtain that both $\alpha_{1}^{(\mathrm{Li})}$ and $\alpha_{1}^{(\mathrm{Mg})}$ are negative and close to zero. In fact, the more negative values are reached for the "zero alloy" composition $\mathrm{Li}_{0.7} \mathrm{Mg}_{0.3}$, where $\alpha_{1}^{(\mathrm{Li})} \approx-0.07$ and $\alpha_{1}^{(\mathrm{Mg})} \approx-0.01$. We also obtain that each $\mathrm{Li}$ atom has an average of 8.5 Li-type NN's, whereas a $\mathrm{Mg}$ atom has around four Mg-type NN's.

Another way of getting insight into the ordering tendencies of the binary alloy can be obtained by resorting to the radial concentration correlation function $\rho_{\mathrm{CC}}(r)$, and this is shown in Fig. 9 for $\mathrm{Li}_{0.7} \mathrm{Mg}_{0.3}$ at $T=875 \mathrm{~K}$, where we plot the $\rho_{\mathrm{CC}}(r)$ obtained by Ruppersberg et al. [4] from their experimental $S(q)$ which clearly indicates, at the NN distance, some preference for heterocoordination. In the figure we have also included both the theoretical and the MD-based $\rho_{\mathrm{CC}}(r)$ at $T=887 \mathrm{~K}$. Moreover, our theoretical results for the other concentrations studied in this work, predict for all concentrations, a weak tendency toward heterocoordination. This behavior can be easily analyzed in terms of the longwavelength limit of the Bathia-Thornton concentrationconcentration partial factor $S_{\mathrm{CC}}(0)$. The usefulness of $S_{\mathrm{CC}}(0)$ in order to obtain microscopic information in a liquid alloy stems from the fact that it can be derived from thermodynamic magnitudes; this is very convenient because of the practical difficulties for measuring, via diffraction experiments, the partial structure factors at low- $q$ values. Now, $S_{\mathrm{CC}}(0)$ can be obtained from

$$
S_{\mathrm{CC}}(0)=N K_{B} T\left(\frac{\partial^{2} G}{\partial x_{1}^{2}}\right)_{N, P, T}^{-1}=\left(1-x_{1}\right)\left(\frac{\partial \ln a_{1}}{\partial x_{1}}\right)_{N, P, T}^{-1},
$$

where $N$ is the total number of particles, $G$ is the Gibbs free energy, and $a_{i}$ and $x_{i}$ are the thermodynamic activity and concentration of component $i$, respectively.

Figure 10 shows, as a function of concentration, the theoretical results obtained for $S_{\mathrm{CC}}(0)$ of liquid $\mathrm{Li}_{x} \mathrm{Mg}_{1-x}$ at $T=887 \mathrm{~K}$. In the figure we have included the ideal mixture result, i.e., $S_{\mathrm{CC}}(0)=x_{\mathrm{Li}} x_{\mathrm{Mg}}$, and also the experimental data derived, according to Eq. (16), from the activity measurements of Saboungi and Blander [61]. The experimental data clearly indicate that the ions in the $\mathrm{Li}_{x} \mathrm{Mg}_{1-x}$ liquid alloy, show, within the range $0.2 \leqslant x_{\mathrm{Li}} \leqslant 0.9$, a slight preference for heterocoordination; in other words, there is a mild

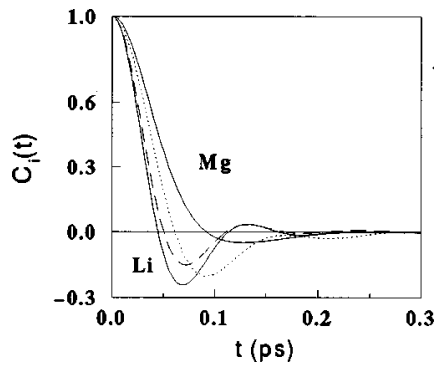

FIG. 11. Molecular dynamics normalized velocity autocorrelation functions $C_{\mathrm{Li}}(t)$ and $C_{\mathrm{Mg}}(t)$, in the $\mathrm{Li}_{0.7} \mathrm{Mg}_{0.3}$, liquid alloy at $T=887 \mathrm{~K}$. For comparison, we have also included the MD results for $C_{\mathrm{Li}}(t)$ of pure $\mathrm{Li}$ at $T=843 \mathrm{~K}$ (dashed line) and those for $C_{\mathrm{Mg}}(t)$ for pure $\mathrm{Mg}$ at $T=953 \mathrm{~K}$ (dotted line).

compound-forming tendency. In fact, this is the behavior predicted by the present NPA-based theoretical results which closely follow the experimental data.

\section{Dynamic properties}

We have also performed MD calculations for the zero alloy composition, i.e., $\mathrm{Li}_{0.7} \mathrm{Mg}_{0.3}$, at $T=887 \mathrm{~K}$, and Fig. 11 shows the obtained velocity autocorrelation functions $C_{\mathrm{Li}}(t)$ and $C_{\mathrm{Mg}}(t)$ associated with the screened $\mathrm{Li}$ and $\mathrm{Mg}$ ions in the alloy. Again, we observe that as a consequence of the smaller mass of the $\mathrm{Li}$ ion, the minimum of $C_{\mathrm{Li}}(t)$ is deeper and appears at shorter times. Now, either by integrating $C_{i}(t)$ or from the slope of the mean square displacements, the corresponding self-diffusion coefficients are readily obtained; these are (in $10^{-5} \mathrm{~cm}^{2} / \mathrm{s}$ units) $D_{\mathrm{Li}}=17.5$ and $D_{\mathrm{Mg}}=12.0$, where the higher value is associated to the lighter and smaller atoms $(\mathrm{Li})$.

At this stage, it is interesting to compare these results with those obtained for the pure components at similar thermodynamic states. By using the NPA-based interatomic pair potentials, MD simulations have been performed for both pure $\mathrm{Li}$ at $T=843 \mathrm{~K}$ and $\rho=0.0416 \AA^{-3}[62]$ and pure $\mathrm{Mg}$ at $T=953 \mathrm{~K}$ and $\rho=0.0383 \AA^{-3}$ [63], and in Fig. 11 we also include the corresponding velocity autocorrelation functions.

First, in the case of $\mathrm{Li}$ it is observed that $C_{\mathrm{Li}}(t)$ in the alloy is steeper, and also has a deeper and wider minimum than $C_{\mathrm{Li}}(t)$ for the pure system. This behavior reflects the fact that the backscattering effects, related to the collisions between a $\mathrm{Li}$ ion and its $\mathrm{NN}$, are stronger in the alloy, where a $\mathrm{Li}$ ion is surrounded by an average of $8.5 \mathrm{Li}$ ions and four (heavier) $\mathrm{Mg}$ ions, than in the pure system where the $\mathrm{Li}$ ion is surrounded by $13 \mathrm{Li}$ ions. As a consequence, the MD result for the self-diffusion coefficient in the pure system $\left(D_{\mathrm{Li}}=24.7\right.$ whereas the experimental value is 25.8 [64]) is substantially larger than the corresponding value in the alloy. Now, in the case of $\mathrm{Mg}$, the above mentioned effects are much stronger, as reflected in the corresponding $C_{\mathrm{Mg}}(t)$. Whereas in the pure system a $\mathrm{Mg}$ ion is surrounded by an average of $11.5 \mathrm{Mg}$ ions, when it comes to the alloy is has an average of four $\mathrm{Mg}$ ions and 9.5 (lighter) Li ions; therefore, the backscattering effects in the alloy are strongly reduced, leading to a $D_{\mathrm{Mg}}$ which is much larger than the value obtained for the pure system $\left(D_{\mathrm{Mg}}=6.7\right)$. 


\section{CONCLUSIONS}

In this work, we have studied the structural and ordering properties of two liquid alloys, i.e., Li-Na and $\mathrm{Li}-\mathrm{Mg}$, which, according to the experimental data, exhibit rather different ordering tendencies. The approach followed in these calculations consists of a combination of the NPA method to obtain the interatomic pair potentials with the MHNC-REP theory of liquids to obtain the static structure, leading to a whole theoretical framework which is free of adjustable parameters, requiring the atomic numbers and the thermodynamic state under study as the only input data. Furthermore, the present study has been completed by performing, for some specific concentrations, MD simulations which, besides complementing the theoretical calculations, also give information on some dynamical properties.

Concerning the Li-Na liquid alloy, the obtained theoretical results for the static structural properties show a rather good agreement with the available experimental data. In fact, we stress that, although during the last years several theoretical works have already been devoted to a study of the liquid Li-Na alloy at the zero alloy composition $[1,16]$, the present results are, to our knowledge, the first theoretical calculations which quantitatively yield both a high $S(q \rightarrow 0)$ and a splitting of the main peak. On the other hand, the MD simulations suggest that the NPA effective interatomic potentials can also provide qualitative information concerning the demixing region.

A similar degree of accuracy has also been achieved in the description of the Li-Mg liquid alloy. This is a system which has not attracted much attention in the past, and the few theoretical works devoted to this liquid alloy have been mainly based on semiempirical models [60,59]. However, the present results show that the ordering tendencies in this alloy can also be well described by resorting to realistic interatomic pair interactions.

We note that although the Li-Na and Li-Mg liquid alloys exhibit opposite ordering tendencies, this work shows that the NPA method is capable of producing reliable effective interatomic pair potentials which can accurately describe the static structural properties in both systems. Therefore, we conclude that the method may be used to derive effective interactions for those metallic systems where the NFE theory holds, and, therefore, the pseudopotential perturbation theory can be applied.

Finally, we point out that the present NPA+MHNC-REP theoretical framework should be considered as a first step in a whole self-consistent approach where the screening charges around the $A$ - and $B$-type ions are computed, not for each ion immersed into a homogeneous electron gas, but taking into account the structure of the alloy and the existence of two different types of ion. Work is in progress, and the results will be reported in due course.

\section{ACKNOWLEDGMENTS}

The work was supported by the SCIENCE Program of the EU (Contract No. ERSCI*-CT910754), the DGICYT (Grant No. PB95-0720-C02-01), and the Junta de Castilla y Leon (Grant No. VA63/96).
[1] W. H. Young, Rep. Prog. Phys. 55, 1769 (1992).

[2] U. Balucani and M. Zoppi, Dynamics of the Liquid State (Oxford University Press, Oxford, 1994).

[3] R. N. Singh, Can. J. Phys. 65, 309 (1987).

[4] H. Ruppersberg, J. Saar, W. Speicher, and P. Heitjans, J. Phys. (Paris), Colloq. 41, C8-595 (1980).

[5] H. Ruppersberg and W. Knoll, Z. Naturforsch. A 32, 1374 (1977).

[6] F. Perrot, Phys. Rev. A 42, 4871 (1990).

[7] F. Perrot and N. H. March, Phys. Rev. A 41, 4521 (1990); 42, 4884 (1990).

[8] L. E. González, D. J. González, M. Silbert, and J. A. Alonso, J. Phys.: Condens. Matter 5, 4283 (1993).

[9] D. J. González, L. E. González, and K. Hoshino, J. Phys.: Condens. Matter 5, 9261 (1993).

[10] L. E. González, A. Meyer, M. P. Iniguez, D. J. González, and M. Silbert, Phys. Rev. E 47, 4120 (1993).

[11] L. E. González, D. J. González, A. Meyer, and M. Silbert, J. Phys.: Condens. Matter 8, 4465 (1996).

[12] Y. Rosenfeld and N. W. Ashcroft, Phys. Rev. A 20, 1208 (1979).

[13] W. Kohn and L. J. Sham, Phys. Rev. A 140A, 1133 (1965).

[14] D. Levesque, J. J. Weis, and G. Chabrier, J. Chem. Phys. 94, 3096 (1991).

[15] H. Mori, K. Hoshino, and M. Watabe, J. Phys.: Condens. Matter 3, 9791 (1991).

[16] H. Mori, K. Hoshino, and M. Watabe, J. Phys. Soc. Jpn. 61, 1218 (1992).
[17] H. Mori, K. Hoshino, and M. Watabe, J. Non-Cryst. Solids 156/158, 85 (1993).

[18] N. W. Ashcroft and D. C. Langreth, Phys. Rev. 159, 500 (1967).

[19] G. E. Bacon, Neutron Diffraction (Clarendon, Oxford, 1975).

[20] A. B. Bhatia and D. E. Thornton, Phys. Rev. B 2, 3004 (1970).

[21] H. Ruppersberg and H. Egger, J. Chem. Phys. 63, 4095 (1975).

[22] H. J. C. Berendsen, J. P. M. Postma, W. F. van Gunsteren, A. Dinola, and J. R. Haak, J. Chem. Phys. 81, 3684 (1984).

[23] N. H. March and M. P. Tosi, Atomic Dynamics in Liquids (Dover, New York, 1991).

[24] F. A. Kanda, R. C. Faxon, and D. V. Keller, Phys. Chem. Liq. 1, 61 (1968).

[25] H. K. Schurmann and R. D. Parks, Phys. Rev. Lett. 27, 1790 (1971).

[26] P. D. Feitsma, J. J. Hallers, F. V. D. Werff, and W. van der Lugt, Physica B \& C 79, 35 (1975).

[27] V. K. Ratti and A. B. Bhatia, Nuovo Cimento B 43, 1 (1978).

[28] K. Hoshino and W. H. Young, J. Phys. F 16, 1671 (1986).

[29] D. J. González and M. Silbert, J. Phys. F 18, 2353 (1988).

[30] J. Hafner and W. Jank, J. Phys. F 18, 333 (1988).

[31] D. Stroud, Phys. Rev. B 9, 396 (1974).

[32] N. W. Ashcroft and D. Stroud, Solid State Phys. 33, 1 (1978).

[33] K. Hoshino, M. Silbert, A. Stafford, and W. H. Young, J. Phys. F 17, L49 (1987).

[34] K. Hoshino and J. J. van Wering, J. Phys. F 18, L23 (1988).

[35] N. W. Ashcroft, Phys. Lett. 23, 48 (1966). 
[36] G. B. Bachelet, D. R. Hamann, and M. Schluter, Phys. Rev. B 26, 4199 (1982).

[37] M. Hasegawa, K. Hoshino, M. Watabe, and H. W. Young, J. Non-Cryst. Solids 117\&118, 300 (1990).

[38] J. Jost, D. Heydt, J. Spehr, and H. Ruppersberg, J. Phys.: Condens. Matter 6, 321 (1994).

[39] B. E. Warren, X-Ray Diffraction (Addison-Wesley, Reading, MA, 1969).

[40] J. M. Cowley, Phys. Rev. 77, 667 (1950).

[41] R. N. Singh and F. Sommer, Rep. Prog. Phys. 60, 57 (1997).

[42] R. L. McGreevy, A. Baranyai, and I. Ruff, Phys. Chem. Liq. 16, 47 (1986).

[43] K. Nakanishi, S. Okazaki, K. Ikari, T. Iguchi, and H. Tanaka, J. Chem. Phys. 76, 629 (1982).

[44] M. Schoen and C. Hoheisel, Mol. Phys. 53, 1367 (1984).

[45] P. Borgelt, C. Hoheisel, and G. Stell, J. Chem. Phys. 92, 6161 (1990).

[46] C. Rey, L. J. Gallego, L. E. González, and D. J. González, J. Chem. Phys. 97, 5121 (1992).

[47] M. C. Abramo, C. Cacamo, and G. Giunta, Phys. Rev. A 34, 3279 (1986).

[48] C. Hoheisel and F. Kohler, Fluid Phase Equilibria 16, 13 (1984)

[49] L. L. Lee, T. H. Chung, and K. E. Starling, Fluid Phase Equilibria 12, 105 (1983).

[50] R. G. Rubio, M. G. Prolongo, M. Diaz-Peña, and J. A. R. Renuncio, J. Phys. Chem. 91, 1177 (1987).
[51] J. A. Padró, M. Canales, G. Sesé, and A. Giró, Physica A 148, 253 (1988).

[52] J. P. Hansen and I. R. McDonald, Theory of Simple Liquids (Academic, London, 1986).

[53] J. Casas, L. E. González, and D. J. González (unpublished).

[54] P. Beauchamp, R. Taylor, and V. Vitek, J. Phys. F 5, 2017 (1975).

[55] L. Dagens, M. Rasolt, and R. Taylor, Phys. Rev. B 11, 2726 (1975).

[56] J. Hafner, J. Phys. F 6, 1243 (1976).

[57] J. Hafner, Phys. Rev. A 16, 351 (1977).

[58] W. A. Harrison, Pseudopotentials in the Theory of Metals (Benjamin, New York, 1966).

[59] J. Hafner, A. Pasturel, and P. Hicter, J. Phys. F 14, 1137 (1984).

[60] B. Karaoglu and W. H. Young, Phys. Chem. Liq. 24, 43 (1991).

[61] M. L. Saboungi and M. Blander, J. Electrochem. Soc. 122, 1631 (1975).

[62] M. Canales, L. E. González, and J. A. Padró, Phys. Rev. E 50, 3656 (1994).

[63] M. M. G. Alemany, J. Casas, C. Rey, L. E. González, and L. J. Gallego, Phys. Rev. E 56, 6818 (1997).

[64] M. Gerl and A. Bruson, in Handbook of Thermodynamic and Transport Properties of Alkali Metals, edited by R. W. Ohse (Blackwell, Oxford, 1985), Chap. 7.5. 